\title{
IL CERVELLO E LA PSICOLOGIA ROMANTICA
}

\author{
Stefano POGGI ${ }^{1}$
}

- RESUMO: Trata-se de uma investigação histórica da neurologia alemã das primeiras décadas do século XIX, representada por Burdach e Carus, e da relação que manteve com pressupostos românticos de abordagem biológica. Carus acentua a necessidade de pesquisar o cérebro pelo viés anatômico e o considera um organismo total, atestando a influência do filósofo Schelling. Burdach, por seu lado, investiga a estrutura cerebral em conexão com a sensibilidade e o processo evolutivo.

- PALAVRAS-CHAVE: Ciência; romantismo; neurologia; psicologia.

1 Un esame di alcuni aspetti della indagine neurologica della Germania dei primi due decenni del secolo XIX, può presentare aspetti assai interessanti. La neurologia mostra infatti di svolgere un ruolo rilevante nello sviluppo dell'interesse dell'età romantica per il problema del vivente: è anzi proprio nelle indagini intorno alla struttura ed alle funzioni del cervello che viene a trovare una delle sue espressioni più efficaci il tipico nesso romantico tra ricerca empirica e speculazione filosofica (Poggi, 1988; Mann \& Dumont, 1988). E le figure più importanti, in questa prospettiva, sono Karl Fr. Burdach (1819-1827) e Carl. G. Carus (1814) (Su Carus: Feremutsch, 1951; su Burdach: Neuburger, 1896, p.310-3).

Volgiamo innanzitutto la nostra attenzione a Carus. Nel suo Versuch einer Darstellung des Nervensystems (1814), Carus svolgeva una inda-

1 Universidade de Florença - Itália. 
gine a due livelli: un livello empirico-osservativo ed uno invece di carattere più teorico. In primo luogo Carus affrontava la questione del modo in cui mostra di essere costituita la "massa nervosa". La "sostanza nervosa" è formata da una quantità di "punti materiali", che costituiscono una "massa puntiforme" che ha una struttura paragonabile a quella di un cristallo ed all'interno della quale sono all'opera delle "forze polari" (Carus, 1814, p.54-6).

Carus respingeva peraltro con decisione il materialismo di Priestley e di Bonnet, dichiarandosi espressamente contro ogni ipotesi di riduzione della attività nervosa al piano delle manifestazioni della psiche. Ma, nello stesso tempo, Carus teneva a sottolineare l'esistenza di un nesso profondo tra l'attività nervosa e i fenomeni elettrici e luminosi: per Carus - come per tutta la ricerca scientifica dell'età romantica in Germania - le scoperte e le ipotesi del "galvanismo" avevano una importanza fondamentale. Da ciò discendeva in Carus la convinzione che il sistema nervoso dovesse essere preso in considerazione non solo dal punto di vista funzionale, ma anche da quello anatomico. Proprio l'osservazione anatomica imponeva di riconoscere che il sistema nervoso presenta una struttura fondamentalmente simmetrica: su tale base, Carus riteneva possibile indicare nei gangli nervosi i "poli" ovvero i "centri di forza" dove si incrociano le fibre nervose e dove l'attività nervosa si concentra, per poi irraggiarsi nei vari organi (Ibidem, p.42-7).

È del tutto evidente l'ispirazione schelinghiana di una visione di questo genere, e non v'è dubbio che le idee di Schelling sull'uomo e sulla sua collocazione nella natura esercitavano una profonda influenza su Carus, che riteneva appunto che l'esistenza umana dovesse essere considerata "la manifestazione di una attività originaria", e appunto di una "attività originaria" che esercita sempre una azione "polare" e che, proprio in virtù di questo suo carattere, supera ed elimina ogni "scissione". Nell'Io dell'uomo doveva essere ravvisata la "più completa manifestazione della aspirazione individualizzante della natura": proprio nel momento in cui noi abbiamo notizia di noi stessi come corpi che hanno sensazioni, noi abbiamo anche notizia di una scissione, di una contrapposizione, di quella che è anzi la prima delle "contrapposizioni da noi percepibili", e cioé di quella tra il nostro Io e il mondo esterno. È così la tensione tra 1'“interno" e l'"esterno" a costituire il primo, fondamentale carattere della attività nervosa, attività nervosa che deve sempre essere considerata una "forza vitale". "Forza vitale" di tipo particolare, che ha in un certo senso il suo "luogo" nel sistema nervoso e che costituisce quindi il nucleo originario della intera attività del corpo. Per potere attua- 
re le sue operazioni, il sistema nervoso necessita d'altronde dell'aiuto di tutti gli altri "sistemi dell'organismo animale", che assolvono quindi alla funzione di un "ponte" tra il sistema nervoso (che è da considerare quindi come l' "interno" dell'organismo) e ciò che, invece, è lo "assolutamente esterno". Da ciò discende che tutti gli altri "sistemi dell'organismo animale" limitano comunque la libertà della attività nervosa, anche se è indubbio che è solo il sistema nervoso ad essere in grado di costituire e di mantenere una effettiva unità dell'organismo (Ibidem, p.11, 40, 102 ss.).

2 Sulla base di questo schema generale, Carus procedeva ad operare una serie di distinzioni tra i vari settori del sistema nervoso, mettendo in primo luogo in evidenza la necessità di distinguere con precisione tra midollo spinale e cervello. Uniformandosi all'opinione della neurologia del tempo, Carus assegnava al primo il controllo sui movimenti del corpo, riservando all'encefalo la capacità di avere sensazioni, in poche parole la sensibilità. In ogni caso, a Carus appariva assai problematico arrivare a individuare un "luogo" preciso per ogni singola funzione del sistema nervoso. Carus era fermamente dell'avviso che ogni tentativo di individuare un "luogo" ovvero un "organo" dell'anima muoveva da presupposti sbagliati. Il sistema nervoso deve essere considerato sempre come una totalità: il sistema nervoso (cioé, più precisamente, il midollo spinale assieme all'encefalo) era considerato da Carus come una sorta di organismo dotato di vita autonoma, in grado di orientare e condizionare l'intero funzionamento del corpo (Ibidem, p.167 ss., 41, 301, 303).

Nel dichiararsi contro l'assunzione di un punto di vista unicamente anatomico nello studio del cervello, Carus teneva a sottolineare inoltre la fondamentale ambiguità di ogni indagine condotta con tecniche lobotomiche e si dichiarava nel contempo a favore delle concezioni di J. C. Reil, uno dei più importanti fisiologi e neurologi dell'epoca (Carus, 1814, p.305. Su Reil: Schott, 1988). Senza per questo assumere posizioni che in qualche modo potessero presentarsi come "spiritualistiche", Reil aveva peraltro sottolineato l'impossibilità di arrivare a dimostrare l'esistenza di un nesso specifico e costante tra le funzioni cerebrali e certe "forme fisse" della "massa cerebrale", ovverosia alcune determinate aree dell'encefalo. Carus condivideva appieno la posizione di Reil, rilevando nel contempo la particolare funzione svolta dal sistema nervoso, e cioé di porre in connessione reciproca tutti gli organi del corpo. Il sistema nervoso ha l'aspetto come di un "fascio di raggi", e ognuno di questi "raggi" da una parte si proietta verso l' "esterno", dall'altra muove nella direzione dello "interno" dell'intero organismo. Questi "raggi" si incrociano e si 
intrecciano in continuazione ed in modi sempre diversi: quella che noi indichiamo come la funzione della "sensibilità" può così essere considerata una sorta di "fuoco" ottico, nel quale la luogo la "diffrazione" dei "raggi" costituiti dalle "fibre nervose". Vi è anzi una continua corrispondenza tra la "massa centrale" del cervello e le terminazioni perifiche del sistema nervoso nei vari "gangli", tanto che si può arrivare a sostenere era questa l'opinione di Carus - che la "massa nervosa centrale" può essere considerata come una "ripetizione", nelle strutture così assunte dalla "sostanza nervosa", dell' "intero organismo specificatamente animale" in tutta la varietà delle sue articolazioni (Carus, 1814, p.308-9).

3 Carus riteneva dunque che non solo fosse legittimo, ma necessario impostare in termini genetici lo studio dell'intero sistema nervoso. E, nello stesso tempo, il suo atteggiamento decisamente polemico nei confronti di un uso sistematico del bisturi nelle indagini sul sistema nervoso (e sull'encefalo in particolare) si univa al riconoscimento dell'importanza assolutamente fondamentale della anatomia comparata. Carus affermava dunque che è solo l'esame delle modificazioni di un organo quello che ci dà la garanzia di coglierne il vero "concetto". È quindi necessario prendere in considerazione e valutare le "modifiche della massa nervosa centrale" $e$, in termini più generali, esaminare lo sviluppo di questa "massa centrale", cioé del cervello, nel "mondo animale", in quello che va considerato come il "perenne, immutabile feto dell'umanità" (Ibidem, p.3).

In Carus prendeva così corpo l'idea di mettere mano a quella che poteva apparire una vera e propria "storia evolutiva" della "massa nervosa centrale". Una "storia evolutiva" di questo genere si sarebbe dovuta fondare sulla assunzione della "idee di un tipo originario di organo centrale della sensibilità". Anche in questo caso Carus mostrava di essere assai vicino ad alcune delle idee di Schelling, anzi - almeno per alcuni aspetti - di dipendenderne, come era dimostrato dal modo in cui Carus formulava la "legge di natura" che mostra di presiedere allo sviluppo di ogni attività dispiegata dall'organismo vivente: "ogni formazione di livello più elevato accoglie in sé stessa quelle che si trovano ai livelli inferiori, e le replica in sé stessa". In forza di questa legge, l'esame del sistema nervoso deve indagare in primo luogo le "manifestazioni della forza dell'anima" che si danno ai livelli inferiore del regno animale, dato che in ogni caso le "più nobili" di tali "manifestazioni" costituiscono solo il punto d'arrivo di un processo evolutivo lungo e complicato (Ibidem, p.13, 43, 15-7, 72-4, 299 ss.). 
Tra l'organismo "psichico" e l'organismo "somatico" non si dà, in realtà, una linea di separazione ben definita: anche ciò che è "organicosomatico" si mostra capace di un processo di sviluppo tale da pervenire ad un livello superiore, tale da presentare forme "più elevate". Il "senso" affermava Carus - è la "base della facoltà psichica superiore", ed in esso non è difficile ravvisare una "aspirazione ideale". Nella attività sensoriale accade dunque che viene a compiersi una sorta di vera e propria "ripetizione" di quella "pulsione di appropriazione" originaria che è all'opera già nelle forme inferiori del vivente: è la contrapposizione - una contrapposizione polare - tra il "senso esterno", il "senso della pelle" e il "senso interno", il "senso delle interiora" che costituisce il primo, decisivo livello di quella tensione tra un "esterno" e un "interno" che, in realtà, costituisce il tratto costitutivo fondamentale della vita psichica nella sua totalità.

4 Tuttavia, il Versuch einer Darstellung des Nervensystems di Carus non può essere considerata un'opera originale. In realtà, il Versuch di Carus era profondamente influenzato dalle idee di Burdach, anche se è vero che il primo volume del ponderoso trattatto dedicato da Burdach allo studio della anatomia e delle funzioni del cervello (Vom Baue und Leben des Gehirns) veniva pubblicato solo cinque anni dopo il Versuch di Carus, e cioé nel 1819. Sarebbero poi seguiti altri due volumi, rispettivamente nel 1822 e nel 1827. Il fatto è che Carus era stato avviato allo studio della neurologia proprio da Burdach, negli anni dei suoi studi universitari a Lipsia.

Le considerazioni che Burdach svolgeva nel terzo volume del suo trattato presentano un interesse tutto particolare. Burdach metteva in rilievo il ruolo centrale che la sensibilità svolge nell' intera vita dell'organismo e procedeva poi ad una esposizione complessiva della sua concezione del sistema nervoso. Come già emerso in Carus, era la tesi secondo cui la sensazione da una parte e il movimento dall'altra costituiscono una sorta di articolazione dell'“anima" verso l'esterno, delle vere e proprie "membra" dell" "anima" ad essere considerata fondamentale. Agli organi della sensazione e del movimento viene così assegnato un "luogo" alla periferia del corpo, mentre 1" interno" di quest'ultimo è in certo senso occupato dal cervello, e cioé a dire dall'organo che funge da mediatore nelle contrapposizioni che si istituiscono tra tutti gli organi (ovvero, più precisamente, tra le funzioni che da essi sono esplicate), contrapposizioni che in ultima analisi vengono eliminate dalla armonia che il cervello assicura all'esplicarsi di tali funzioni medesime. L'esistenza di un "sensorium commune" - di quello che può essere considerato come un "sesto 
senso" - costituisce il modo in cui il ruolo di mediazione e di compensazione svolto dal cervello si documenta con la massima chiarezza e incisività. D'altro canto, da un punto di vista generale, è senz'altro possibile considerare tutti gli organi di senso come altrettante "vie di accesso alla natura dell'anima" (Burdach, 1819-1827, v.3, p.211-3).

Tra gli stimoli sensoriali e le reazioni sensoriali, tra il mondo esterno e il mondo interno si dispiega una interazione continua, che fa sì che ogni organo presenti la medesima "struttura fondamentale". Come già in Carus (ma il Versuch di quest'ultimo era in realtà nient'altro che una esposizione delle idee di Burdach), gli organi della sensibilità venivano concepiti da Burdach come "organi di appropriazione". Anzi, Burdach teneva a sottolineare come l'indagine anatomo-morfologica avesse fornito la prova più chiara ed esplicita del fatto che ogni organo di senso costituisce il risultato finale di un processo di sviluppo e trasformazione della epidermide. In altri termini: l'epidermide - che costituisce la prima, sottile linea di divisione tra l" "esterno" e l' "interno", tra il mondo e l'organismo, attraversa una serie di trasformazioni, un vero e proprio processo evolutivo che conduce ai diversi organi di senso, tutti accomunati da una medesima "struttura fondamentale" (Ibidem, p.215-22).

5 Non v'è dubbio che, tra tutti gli organi di senso, è il cervello ad assolvere ad una funzione centrale. Il cervello si presenta infatti come organo di coordinazione di tutte le cosiddette "forze psichiche", di tutte le funzioni degli organi di senso. Ed alle "forze psichiche" si deve guardare negli stessi termini in cui si parla di una "forza vitale". La "forza vitale" - affermava in sostanza Burdach - è un termine del quale non facciamo certamente mai uso per designare delle specifiche operazioni del vivente. Anche chi è convinto della esistenza di una "forza vitale" non ritiene certo che essa costituisca la condizione delle specifiche operazioni di uno specifico organo. Al contrario, la "forza vitale" si manifesta sotto le forme più diverse, ed è solo perché tutti i suoi vari modi di manifestarsi hanno in realtà un unico fondamento, che la vita può arrivare al suo completo sviluppo, può presentarsi come la vita di un organismo nella sua completezza. Ciò che vale per la "forza vitale", non può dunque non valere per l'attività dei sensi, attività che si estrinseca come "forza psichica": ed è solo per via di astrazione che è possibile distinguere tra tipi diversi di attività sensoriale. Anzi, si può dire che in realtà non è possibile operare delle distinzioni nette tra i vari tipi di attività dei sensi, dato che la natura ci offre una grande quantità di prove concrete della unità di fondo dell'anima. E a tale situazione corrisponde il fatto che l'organo 
della attività dei sensi - il sistema nervoso - si presenta alla osservazione come un sistema di elevata complessità e, nello stesso tempo, contraddistinto da un elevato livello di interconnessione (Ibidem, p.266-9).

Come già aveva fatto Carus nel 1814, ancora nel 1827 Burdach negava la possibilità di procedere ad una netta distinzione tra le funzioni vitali inferiori da un lato e, dall'altro, le funzioni del sistema nervoso. Gli organi di senso debbono essere considerati una sorta di "riflessi corporei della attività dell'anima"; e la vita dell'anima trova la sua concreta effettiva estrinsecazione nella attività cerebrale. Nello stesso tempo, l'anima trova nel corpo una sorta di "contrappeso" che "inibisce" le sue operazioni: ogni mutamento di stato psichico deve essere accompagnato da un mutamento corrispondente nei processi degli organi interni. In una qualche misura, l'attività del sistema nervoso deve essere considerata come il vero e proprio compimento della vita ideale dell'anima: è necessario infatti riconoscere che l'attività del sistema nervoso dell'uomo perviene a livelli sempre più articolati e sviluppati, raggiungendo una complessità di struttura che proprio in quanto tale costituisce il fondamento della individualità e della personalità (Ibidem, p.193-5).

6 Le concezioni di Burdach - e non solo le sue concezioni relative alle funzioni cerebrali - muovevano dalla assunzione di un punto di vista morfologico e genetico. Allorché aveva sostenuto la necessità di mettere mano ad una descrizione del processo di sviluppo della "massa nervosa centrale", Carus si era in realtà ricollegato alle idee di Burdach, che già nella sua prolusione Über die Aufgabe der Morphologie del 1817 aveva illustrato in modo esauriente caratteri e prospettive di una indagine di tipo genetico-evolutivo (Burdach, 1817).

La messa in atto e la realizzazione coerente di questo tipo di indagine erano destinate nel giro di pochi anni a dare molti e importanti frutti, e tra questi un posto di primissimo rilievo è quello occupato da uno dei primi documenti della embriologia moderna, e cioé dallo Über die Entwicklungsgeschichte der Thiere (1828-1837) di K. E. von Baer. Su von Baer (Rajkov, 1968; Oppenheimer, 1967). Le ricerche e le teorie del fondatore della embriologia moderna sono state a più riprese esaminate $\mathrm{e}$ discusse: tuttavia, non si può non lamentare il fatto che non si sia dedicata tutta la necessaria attenzione alla importanza che von Baer assegnava allo studio del processo di formazione del sistema nervoso nei vertebrati. È infatti proprio questo processo che ha una importanza centrale nella concezione di von Baer. L'esame del processo di formazione degli organi nel corso della crescita dell'organismo dei vertebrati conduceva infatti von Baer alla constatazione di un primo fatto di ordine generale e 
di importanza fondamentale, e cioé che l'embrione dei vertebrati consiste inizialmente di una sorta di "tubuli", "uno superiore per la metà costituita dal tronco, uno inferiore per quella costituita dall'addome", mentre la colonna vertebrale costituisce in un certo senso l' "asse comune" di tali "tubuli" (Baer, 1828-1837, v.1, p.153 ss.).

L'esame comparato dei diversi stadi di formazione dell'embrione non può non mettere in luce l'importanza della collocazione centrale che, in tale sviluppo, viene ad essere assunta dalla colonna vertebrale. "Noi possiamo - precisava von Baer - paragonare, se consideriamo l'embrione nella sua sezione sagittale, lo schema secondo cui i verterbrati si sviluppano nel loro processo di crescita con un 8". È infatti del tutto evidente che nel loro crescere, i due "tubuli fondamentali" del torace e dell'addome, disegnano, nel loro condividere l' "asse comune" della colonna vertrebrale, una figura che è quella del numero 8 , fermo ovviamente restando che poi, nel corso della maturazione dell'embrione, muta la disposizione della colonna vertebrale nei confronti della cassa toracica e dell'addome (Ibidem, p.164).

7 È nello stesso tempo ovvio anche che ognuno dei due "tubuli principali" subisce un ulteriore processo di suddivisione in una serie di "foglietti", a loro volta destinati a dare luogo, nel ripiegarsi su sé stessi, a nuovi "tubuli". Sono questi ultimi a dovere essere considerati come "organi fondamentali", e cioé quegli "organi fondamentali" a partire dai quali "sono gli specifici organi a prendere gradualmente forma".

Se concentriamo dunque la nostra attenzone su ciò che sembra caratterizzare in modo specifico la formazione e lo sviluppo del sistema nervoso, è innanzitutto un dato osservativo che deve essere sottolineato. Ciò che risulta con tutta chiarezza nella esposizione di von Baer è che da una parte l'epidermide (una sorta di "tubulo generale esterno", che abbraccia e avvolge le due metà in cui si divide l'embrione, la cui sezione sagittale ha appunto l'aspetto del numero 8), dall'altra la colonna vertebrale hanno comunque la loro comune radice nel "germe" dell'embrione. Tracciare una netta linea di demarcazione tra l'epidermide e i cosiddetti "tubuli nervosi" è possibile solo sulla base di una preliminare e più fondamentale distinzione tra i diversi organi (Ibidem, p.153 ss.).

Non ci si deve lasciare sfuggire l'importanza del rapporto così messo in luce da von Baer tra l'epidermide e la colonna vertebrale. A tale proposito è possibile senz'altro richiamarsi alle teorie di Oken (ed alla lunga controversia di quest'ultimo con Goethe); ma ciò che mostra di essere di particolare interesse è soprattutto il significato che von Baer assegnava a quello che egli chiamava l'"accordo orginario" tra l'epidermide e la colon- 
na vertebrale, nel quadro di una generale attenzione per lo studio dello sviluppo degli organismi in generale e non solo quindi dei vertebrati.

L'assunzione di un punto di vista di tipo genetico-evolutivo da parte di von Baer mostrava di porre le basi di una spiegazione di carattere complessivo del processo nei cui termini nasce e si sviluppa la vita psichica. "L'accordo originario" della epidermide e del midollo spinale sembrava così di grande importanza.

Se l'epidermide costituisce la periferia e il secondo invece il centro del sistema nervoso animale, possiamo allora constatare - affermava von Baer - la semplicità del processo con cui una parte di ciò che è originariamente comune viene dislocato verso l'interno via via che si forma il canale del midollo spinale, mentre l'altra rimane nella sua posizione alla periferia. Ed è nella parte che si trova ad essere così racchiusa all'interno che la vita animale si sviluppa sino alla sua massima fioritura, mentre la parte rimasta alla periferia non si innalza al di là di tale livello inferiore. (Baer, 1828-1837, v.1, p.166.)

Altrettanto importante era poi per von Baer porre l'attenzione sulla necessità di distinguere tra il livello di sviluppo raggiunto dal "corpo animale" e il "tipo di organizzazione" che in esso presentano le funzioni vitali e di ravvisare quindi il rapporto che esiste tra tale livello di sviluppo e la maggiore o minore "eterogeneità delle parti elementari o delle singole sezioni di un apparato composto" (Ibidem, p.207). Discendeva da ciò l'esigenza di prendere in esame i "tipi fondamentali" di organizzazione della vita animale che ci è dato di ravvisare in natura. E se come "tipo" di siffatta organizzazione andava inteso il modo in cui si distribuiscono e si pongono in rapporto reciproco gli "elementi organici" e gli organi, per von Baer era anche chiaro che è proprio la grande differenza che esiste tra i diversi "tipi" di sistema nervoso a fornirci i migliori strumenti di orientamento e classificazione (p.208-9). Ma la conseguenza forse più importante della assunzione di questo punto di vista era la constatazione di tutte le ambiguità insite alla denominazione del cervello come "organo dell'anima": "se con l'espressione di cervello intendiamo designare - affermava von Baer - non un organo determinato, ma la parte centrale del sistema nervoso o la massa di nervi che riceve le impressioni sensoriali, allora si può senz'altro attribuire un cervello agli insetti” (p.235-6).

8 Era così proprio una delle tesi fondamentali di Burdach - e cioé la tesi secondo cui "la legge generale del processo di sviluppo della vita [è] quella della subordinazione della plasticità alla sensibilità" (Burdach, 1819-1827, v.3, p.188) - a trovare più che una eco nella concezione di von 
Baer. E occorre inoltre sottolineare, in questo quadro, il fatto che von Baer teneva a porre in particolare rilievo l'esistenza di una connessione costante tra le funzioni della nutrizione e quelle della sensazione.

Anche il fondatore di una teoria così come controversa come la cosiddetta "cranioscopia" - F. J. Gall - aveva sostenuto la tesi secondo cui gli organi di senso devono essere considerati "organi di appropriazione". Ma, nondimeno, Gall aveva anche preso una posizione duramente ciritica nei confronti delle concezioni di Carus e di Burdach. In primo luogo, Gall criticava quella che gli appariva una frettolosa e superficiale generalizzazione di un piccolo numero di dati osservativi (Gall \& Spurzheim 1809 , p.16, 62, 405). Gall nutriva invece la ferma convinzione che non esistessero dei buoni motivi per sostenere l'omogeneità strutturale delle fibre nervose, e su questa linea si schierava con decisione contro quei fisiologi (non erano Carus e Burdach, ma soprattutto Rudolphi ad essere oggetto delle sue dure riserve) che avevano sostenuto la tesi della localizzazione centrale delle funzioni nervose. I presupposti speculativi (quando non spiritualistici) di una concezione di questo tipo erano fin troppo chiari e - ed è l'aspetto più importante della questione - i dati osservativi a disposizione erano assolutamente insufficienti alla conferma di una ipotesi del genere (Gall, 1825, p.50, 7, 125, 262).

Non per questo Gall negava l'unità funzionale del sistema nervoso; egli stesso aveva in realtà compiuto una grande quantità di osservazioni sul piano dello studio anatomico del sistema nervoso che potevano senz'altro valere come conferma di tale unità. Ma ciò che egli contestava con decisione era la tesi secondo cui il cervello deve essere considerato il centro dell'intero sistema nervoso, una sorta di nucleo costitutivo di un apparato di grande complessità e dalla grande ricchezza di articolazioni. Per Gall, l'evidente dipendenza di una tesi del genere dalla idea della semplicità (e della immortalità) dell'anima costituiva proprio un motivo più che sufficiente per respingerla con energia.

Alcune delle idee di Baer non erano molto diverse da quelle che Gall avanzava prendendo di fatto le distanze da Burdach e da Carus. Von Baer non si era infatti dichiarato per la tesi della centralizzazione del sistema nervoso, e l'accento che egli poneva sulla necessità di una netta distinzione tra i diversi tipi di sistema nervoso era un chiaro segno del fatto che egli era profondamente convinto della necessità di procedere con molta cautela nell'esame delle funzioni e della struttura di un organo come il cervello. Quanto poco sopra abbiamo riportato delle convinzioni di von Baer circa il "cervello" degli insetti ce lo conferma in modo inequivocabile (Baer, 1828-1837, v.1, p.209 ss. e 234 ss.) 
È certo difficile passare sotto silenzio i molti rapporti che legano von Baer a Burdach ed alla indagine romantica sul vivente in generale. Ma la distanza di von Baer (e di Gall) dalla unione (quando non dalla commistione) romantica di antropologia (e di cosmologia) e di indagine sul sistema nervoso non può essere in ogni caso occultata. E si tratta di una distanza sul piano metodologico che deve in realtà essere considerata il segno - e segno chiarissimo - dei grandi mutamenti in corso nell'indagine tedesca sul vivente, al chiudersi del periodo di massima fioritura della scienza romantica.

9 L'inizio degli anni 30 del secolo XIX, vede una forte diminuzione dell'interesse della biologia e della fisiologia tedesca per lo studio della neurologia. L'indagine sul sistema nervoso in generale ed in particolare quella intorno al cervello viene progressivamente trascurata. Come già è stato messo a più riprese in evidenza, il periodo $1830-1870$ è contraddistinto, in Germania, da una situazione quasi completa di stallo nella ricerca in questo campo (Neuburger, 1896; Soury, 1899; Clarke \& Jacyna, 1987), e non può non colpire il contrasto con la situazione del primo quarto di secolo. Anche convinti sostenitori della concezione schellinghiana di una "natura divina" come Carus e Burdach avevano allora riconosciuto l'importanza tutta particolare della anatomia cerebrale, come è del resto provato dalla grande considerazione da loro manifestata per l'aspetto potremmo dire più propriamente "tecnico" delle concezioni di Gall, comunque giudicato valentissimo neuroanatomista. E da un punto di vista più generale $-\mathrm{e}$ ciò vale non solo per von Baer (e Gall), ma anche per Burdach e per Carus - era stata proprio la tesi della esistenza di un intimo nesso tra le funzioni del sistema nervoso da una parte e quelle dell'intero organismo dall'altra a incontrare ampio consenso. Il progresso della neurologia era apparso così strettamente connesso a quello di una scienza generale della vita, di una "biologia", come appunto tale disciplina era stata designata da Burdach già nel 1800 - e dunque prima di Lamarck (Jahn et al., 1985, p.319).

Ma è anche ben noto che lo sviluppo delle "scienze della vita" è sempre più contraddistinto, nella Germania dagli anni " 40 in poi, da una impostazione decisamente meccanicistica. Per quanto riguarda specificatamente lo studio del sistema nervoso, la ricerca prende a limitarsi sempre di più all'esame di quella che in certo senso ne può essere detta la "periferia", mentre sono i progressi della chimica e della fisica che mostrano di consentire una conoscenza puntuale di quelle funzioni del vivente che sembrano suscettibili di misurazione. Fa parte di questa linea di sviluppo della fisiologia sperimentale anche il lavoro intorno alla fisi- 
ologia delle sensazioni, che nel contempo si viene a costituire come premessa decisiva alla nascita della cosiddetta "psicologia scientifica". ${ }^{2}$ Si è soliti indicare nella fisiologia degli organi di senso elaborata da Johannes Müller il punto di inizio del processo di sviluppo scientifico che conduce alla nascita della psicologia scientifica (Lohff, 1990; Hagner, 1992; Poggi, 1992; Lenoir, 1992). Più esattamenete, bisognerebbe chiamare in causa le indagini di fisiologia delle sensazioni condotte nell'ambito della cosiddetta "scuola di Müller" (Turner, 1982) e innanzitutto le indagini di ottica e di acustica dovute a Helmholtz. Lo studio della attività degli organi di senso e quindi della sensazione in generale subisce un profondo rinnovamento: una parte consistente di quella che si è soliti indicare come "attività psichica" mostra di potere essere meglio conosciuta proprio perché divenuta oggetto di analisi esatte e sperimentali. E, nello stesso tempo, accade che proprio in nome delle esigenze di esattezza cui deve comunque soddisfare anche l'indagine sulle funzioni del vivente si rinunci consapevolmente allo studio dei rapporti tra attività sensoriale e attività cerebrale.

Ovviamente, il quadro appena delineato in modo tanto elementare potrebbe essere integrato e migliorato sotto molti aspetti: ciò vale in primo luogo per quanto riguarda la parte che Müller stesso (e non solo la sua "scuola") ha nello svolgimento di questa vicenda, in particolare se si guarda alle componenti vitalistiche della sua concezione della attività sensoriale, concezione che esercita d'altronde una potente influenza sul rinnovamento della psichiatria che si avvia con l'opera di Wilhelm Griesinger (1845). Su Griesinger: Wahrig-Schmidt, 1985; Verwey, 1985. Se, comunque, ci limitiamo allo sviluppo della indagine neurologica, rimane incontestabile un fatto, e precisamente il fatto che gli anni ' 40 vedono, in Germania, una fortissima diminuzione di interesse per questo tipo di indagine. Accade invece che sul piano della psichiatria - abbiamo appena ricordato il nome di Griesinger - siano proprio le conezioni "somatiche" a incontrare molti sostenitori, senza che tutto questo comporti un qualche significativo interesse per lo studio della attività cerebrale. Vi è una generale tendenza a considerare il cervello l'“organo dell'anima", ed in tal modo è proprio la vecchia concezione spiritualistica dell'anima come monade a vivere, quasi inaspettatamente, una sorta di rinascita.

10 Sarà solo con gli anni ' 70 che la ricerca neurologica tornerà nuovamente in primo piano, a seguito di una serie di innovazioni tecniche che (come è il caso dell'uso della stimolazione elettrica per studiare la

2 Sull'argomento: Boring, 1957; Woodward-Ash, 1982; Arens, 1989. 
corteccia cerebrale) conducono ad ampliare in misura assai significativa le nostre conoscenze circa le funzioni cerebrali. Alcune delle idee della neurologia romantica di quasi mezzo secolo prima sembrano così trovare conferma, in primo luogo per quanto riguarda la teoria della esistenza di "fibre di associazione" che si ritiene si intersechino nella corteccia cerebrale. Ma anche il tentativo di delineare un sistema "proiettivo" delle funzioni cerebrali sembra essere l'applicazione di alcune delle vecchie idee romantiche. ${ }^{3}$ Burdach aveva in effetti sostenuto l'esistenza di una connessione specifica e costante tra la "topografia" del cervello e la posizione assunta dal corpo nello spazio (Burdach, 1819-1827, v.1, p.1-10). E va inoltre menzionata la nuova importanza che viene riconosciuta al punto di vista morfologico e genetico, sebbene sia soprattutto alla grande influenza delle idee di Spencer che bisogna guardare anche per quanto concerne il rapporto del corpo con il mondo esterno.

Non c'è in ogni caso niente di esagerato nel sostenere che il rinnovarsi della indagine neurologica nella Germania degli anni '70 può essere considerato una sorta di rinascita di molte idee della medicina romantica, e appunto di quelle idee con cui ci siamo incontrati esaminando le tesi di Carus, di Burdach e poi di von Baer. Erano idee grandi e coraggiose, ma che da un lato la carenza di adeguati mezzi tecnici, dall'altra un timore non sempre confessato per quello che ne era l'intrinseco materialismo avevano finito con il fare tacere. Ma, proprio nell'età romantica, era comunque iniziato un processo di riflessione, di presa di coscienza dei futuri compiti della indagine neurologica. La storia della scienza dei nostri giorni ha il compito di rintracciare i sottili ma tenaci fili che legano la neurologia speculativa di Burdach e di Carus con le teorie localizzazionistiche e proiettive di Meynert, di Wernicke, di Fritsch, di Hitzig, di Flechsig. Non è un lavoro semplice, ma certo importante.

POGGI, S. The brain and the romantic psychology. Trans/Form/Ação (São Paulo), v.23, p.105-119, 2000.

- ABSTRACT: The subject is a historical investigation of German neurology from the first decades of the nineteen century, represented by Burdach and Carus, and its relation to the romantic presuppositions of the biological approach. Carus accentuates the necessity of an anatomical research of the brain and considers a total organism, attesting the influence of Schelling's

3 Oltre alla letteratura citata alla nota 29, vedi Young, 1970. 
philosophy. Burdach, for his part, investigates the cerebral structure in conexion with sensibility and with the evolutionary process.

- KEYWORDS: Science; romanticism; neurology; psychology.

\section{Referências bibliográficas}

ARENS, K. Structures of knowing: psychologies of the nineteenth century. Boston: Dordrecht, 1989.

BAER, K. E. Über die Entwicklungsgeschichte der Thiere. Beobachtung und Reflexion. Königsberg, 1828-1837. 2v.

BORING, E. G. A history of experimental psychology. New York: ApplitonCenturyCrofts, 1957.

BURDACH, K. F. Über die Aufgabe der Morphologie: bey Eröffnung der königlichen anatomischen Anstalt in Königsberg geschrieben und mit Nachrichten über diese anstalt begleitet. Leipzig, 1817.

. Vom Baue und Leben des Gehirns. Leipzig, 1819-1827.

CARUS, C. G. Versuch einer Darstellung des Nervensystems und Insbesondere des Gehirns nach ihrer bedeutung, entwicklung und vollendung. Leipzig, 1814.

CLARKE, E., JACYNA, L. S. Nineteenth century origins of neuroscientific concepts. Berkeley, 1987.

FEREMUTSCH, K. Die Grundzüge der Hirnanatomie bei Carl Gustav Carus (1789-1869). Ein Beitrag zur Geschichte der Medizin und der Naturwissenschaften des beginnenden 19. Jahrhunderts. "Centaurus" 2 (1951), S. 52-85.

GALL, F. J. Revue critique de quelques ouvrages anatomo-physiologiques et exposition d'une nouvelle philosophie des qualités morales et des facultés intellectuelles. Paris, 1825.

GALL, F. J., SPURZHEIM, J. K. Untersuchungen über die Anatomie des Nervensystems überhaupt und des Gehirns insbesondere. Ein dem französischen Institute überreichtes Mémoire. Paris-Straßburg, 1809.

GRIESINGER, W. Die Pathologie und Therapie der psychischen Krankheiten. Stuttgart, 1845.

HAGNER, M. Sinnlichkeit und Sittlichkeit. Spinozas "grenzenlose Uneigennützigkeit" und Johannes Müller. In: HAGNER, M., WAHRIG-SCHMIDT, B. (Org.) Johannes Müller und die Philosophie. Berlin,1992. p.29-44.

JAHN, I., LÖTHER, R., SENGLAUB, K. (Org.) Geschichte der Biologie: Theorien, Methoden, Kurzbiographien. Jena, 1985. 
LENOIR, T. Helmholtz, Müller und die Erziehung der Sinne. In: HAGNER, M., WAHRIG-SCHMIDT, B. (Org.) Johannes Müller und die Philosophie. Berlin, 1992. p.207-22.

LOHFF, B. Die Suche nach der Wissenschaftlichkeit der Physiologie in der Zeit der Romantik. Stuttgart-New York, 1990.

MANN, G., DUMONT, F. (Org.) Gehirn-Nerven-Seele: Anatomie und Physiologie im Umfeld S. Th. Sömmerrings, "Sömmerring-Forschungen”. Stuttgart-New York, 1988. v.3.

NEUBURGER, M. Die historische Entwicklung der Gehrin-und Rückenmarkphysiologie vor Flourens. Stuttgart, 1896.

Johann Christian Reil. Stuttgart, 1913.

OPPENHEIMER, J. Embriology and Naturphilosophie: Goethe and von Baer. In:___ (Org.) Essays in the History of Embriology and Biology. Cambridge, Mass., 1967.

POGGI, S. Mind and brain in medical thought during the romantic period. History and Philosophy of Life Sciences, v.10, p.41-53, 1988.

Goethe, Müller und das Problem der Empfindung. In: HAGNER, M., WAHRIG-SCHMIDT, B. (Org.) Johannes Müller und die Philosophie. Berlin, 1992. p.191-206.

RAJKOV, B. E. Karl Ernst von Baer 1792-1876: Sein Leben und sein Werk. Acta historica Leopoldina (Leipzig), v.5, 1968.

SCHOTT, H. Zum Begriff des Seelenorgans bei Johann Christian Reil (1759-1813). In: GUNTER, M., FRANZ, D. (Org.) Gehirn-Nerven-Seele: Anatomie und Physiologie im Umfeld S. Th. Sömmerrings, "Sömmerring-Forschungen". Stuttgart-New York, 1988. v.1, p.183-210.

SOURY, J. Le système nerveux central. Structure et fonctions: histoire critique des théories et de doctrines. Paris, 1899.

TURNER, S. Helmholtz, sensory physiology and the disciplinary development of german physiology. In: WOODWARD, W., ASH, M. G. (Org.) The problematic science: psychology in nineteenth century thought. New York,1982. p.147-66.

VERWEY, G. Psychiatry in an anthropological and biomedical context: philosophical Presuppositions and implications of German psychiatry. Dordrecht-Boston, 1985.

WAHRIG-SCHMIDT, B. Der junge Griesinger im Spannungsfeld zwischen Philosophie und Physiologie. Tübingen, 1985.

WOODWARD, W., ASH, M. G. (Org.) The problematic science: psychology in nineteenth century thought. New York, 1982.

YOUNG, R. M. Mind, brain and adaptation: nineteenth century cerebral localization and its biological context from Gall to Ferrier. Oxford, 1970. 